Pacific Journal of Mathematics

ON THE TRANSFORMATION OF INTEGRALS IN MEASURE 


\title{
ON THE TRANSFORMATION OF INTEGRALS IN MEASURE SPACE
}

\author{
Robin W. ChaneY
}

One objective of this paper is to prove a formula for the transformation of integrals by means of a change of variable in purely measure-theoretic setting. The classical prototype of such formulas is the one in which the change of variable is effected by an (appropriately differentiable) one-to-one transformation from some subset of Euclidean $n$-space $R^{n}$ onto some other subset of $R^{n}$; the jacobian of the transformation plays a key role here. For the present study the transformation which gives the change of variable is no longer assumed to be one-to-one but it is required to satisfy certain standard conditions relative to the measure spaces at hand.

Some of the results presented in this paper can be summarized informally as follows, Let $T$ be a function from a nonempty set $S$ onto a set $X$, let $\{S, \mathfrak{M}, \mu\}$ and $\{X, \mathfrak{N}, \nu\}$ be measure spaces, and let $\mathfrak{B}$ be a sub- $\sigma$-field of $\mathfrak{M}$. These entities are subjected to certain standard requirements. Within this basic setting is proved a formula which takes the form

$$
\int_{B}(H \circ T) f d \mu=\int_{T^{\prime} B} H^{\prime} W(., B) d \nu ;
$$

in (1), $H$ is some $\mathfrak{N}$-measurable function, $B$ is a set in $\mathfrak{B}, f$ is analogous to the jacobian, and ' $W$ is a function having certain measure-theoretic properties. Indeed ' $W(x, B)$ is intended to "count or weigh" the number of points in $B$ mapped into $x$ by $T$. In this paper certain theorems are proved which reveal in detail the relationship between $f$ and ' $W$.

Rado and Reichelderfer have developed in [5] a "general transformation formula" from which the classical formulas for the transformation of integrals can be derived. In [3] Craft extended this formula and Reichelderfer proved a transformation formula in 4.10 in [6] which extends Craft's result. Reichelderfer's formula applies not merely to Lebesgue integration in $R^{n}$ (as the earlier formulas did) but rather is proved in a measure-theoretic (quasi-topological) setting. Formula (1) is also an extension of Craft's result. The theorem (see 3.1 below) in which (1) is proved neither implies nor is implied by the theorem in 4.10 in [6].

Thus the present paper is somewhat similar to [6] in purpose and spirit. For example, in this paper the concept of "weighing function" (the function ' $W$ in (1) is a weighing function) is a generalization of the concept of multiplicity function discussed in [5]. The corresponding 
role in [6] is played by the "weight function." In $\S 5$, we explore certain relationships between weight functions and weighing functions.

Moreover, in 4.5, we establish a fundamental connection between the functions ' $W$ and $f$ appearing in (1); this theorem has as yet no analogue in the theory developed in [6].

1. Standard hypotheses. The standard hypotheses for the present theory are denoted by A1-A6. These hypotheses are stated below.

A1. $\{\boldsymbol{S}, \mathfrak{M}, \mu\}$ is a $\sigma$-finite, complete, positive measure space.

A2. $\{X, \Re, \nu\}$ is a $\sigma$-finite, complete, positive measure space.

A3. $T$ is a function (transformation) from $\boldsymbol{S}$ onto $\boldsymbol{X}$.

$\mathrm{A} 1, \mathrm{~A} 2$, and $\mathrm{A} 3$ are respectively $\mathrm{H} 1, \mathrm{H} 2$, and $\mathrm{H} 3$ in 1 of [6] except for the notational change made in passing from $\mathrm{H} 2$ to $\mathrm{A} 2$.

A4. $\mathfrak{B}$ is a $\sigma$-field of subsets $B$ of $\boldsymbol{S}$ having the following properties. $\mathfrak{B}$ is a subfamily of $\mathfrak{M}$ and $T \mathfrak{B}=\{T B: B \in \mathfrak{B}\}$ is a subfamily of $\mathfrak{R}$. For each set $M$ in $\mathfrak{M}$ there is a set $B$ in $\mathfrak{B}$ such that $M \subseteq B$ and $\mu B=\mu M$.

It follows at once from $\mathrm{A} 1$ and $\mathrm{A} 4$ that for each set $M$ in $\mathfrak{M}$ there are sets $B_{1}$ and $B_{2}$ in $\mathfrak{B}$ such that $B_{1} \subseteq M \cong B_{2}$ and $\mu B_{1}=\mu B_{2}$.

A5. $\mathfrak{A}$ is a $\sigma$-field of subsets $A$ of $\boldsymbol{X}$ having the following properties. $\mathfrak{A}$ is a subfamily of $\mathfrak{N}$ and $T^{-1} \mathfrak{U}=\left\{T^{-1} A: A \in \mathfrak{A}\right\}$ is a subfamily of $\mathfrak{B}$. For each set $N$ in $\mathfrak{R}$ there is a set $A$ in $\mathfrak{A}$ such that $N \subseteq A$ and $\nu N=\nu A$.

It follows at once from $\mathrm{A} 2$ and $\mathrm{A} 5$ that for each set $N$ in $\mathfrak{N}$ there are sets $A_{1}$ and $A_{2}$ in $\mathfrak{U}$ such that $A_{1} \subseteq N \subseteq A_{2}$ and $\nu A_{1}=\nu A_{2}$.

Definition. Assume A1-A5. Let ' $W$ be a nonnegative, extended real valued function defined on $\boldsymbol{X} \times \mathfrak{B}$. ' $W$ is said to be a weighing function for $T$ if the following conditions are satisfied:

(i) If $B$ is in $\mathfrak{B}$ then ' $W(x, B)=0$ a.e. $\nu$ on $C T B(C T B$ denotes the complement of $T B$ relative to $\boldsymbol{X}$ ).

(ii) If a set $B$ in $\mathfrak{B}$ is the union of a countable number of pairwise disjoint sets $B_{i}$ in $\mathfrak{B}$ then ${ }^{\prime} W(x, B)=\Sigma^{\prime} W\left(x, B_{i}\right)$ a.e. $\nu$ on $X$.

(iii) For each $B$ in $\mathfrak{B}$ the function ' $W(., B$ ) is $\mathfrak{R}$-measurable (on $\boldsymbol{X}$ ).

A6. ' $W$ is a weighing function for $T$.

There will be several weighing functions in certain discussions in the sequel. Every weighing function will be denoted by the basic 
symbol ' $W$ together with some subscript or possibly even a superscript. Thus ' $W,{ }^{\prime} W_{0}$, and ' $W *$ will denote weighing functions for $T$.

2. Bounded variation and absolute continuity. Throughout this section the standard hypotheses A1-A6 are assumed to hold.

Definition 1. For each $B$ in $\mathfrak{B}$ the function ${ }^{\prime} W(., B)$ is $\mathfrak{R}$ measurable; set $W B=\int_{X}{ }_{X} W(x, B) d \nu$. The nonnegative, extended real valued number $W B$ is called the weight attached to $B$ by ' $W$. $W$ denotes the function which associates with each $B$ in $\mathfrak{B}$ the attached weight $W B$.

It follows from $\mathrm{A} 4$ and $\mathrm{A} 6$ that $W B=\int_{T^{\prime}}{ }^{\prime} W(x, B) d \nu$ for each $B$ in $\mathfrak{B}$. Observe that $W$ is a nonnegative, extended real valued measure on $\mathfrak{B}$.

For each weighing function there is the corresponding function which associates with each $B$ in $\mathfrak{B}$ its attached weight. This function will henceforth be denoted by the symbol for the corresponding weighing function with the "prime" removed. Thus $W, W_{0}$, and $W *$ denote the weights induced by the weighing functions ' $W$,' $W_{0}$, and ' $W *$ respectively.

DeFinition 2. The transformation $T$ is said to be of bounded variation with respect to the weights $W$-briefly, $T$ is $B V W$-if $W S$ is finite.

Hence $T$ is $B V W$ if and only if each function ' $W(., B)$ is $\nu$ integrable over $\boldsymbol{X}$.

Definition 3. Let $f$ be a nonnegative, extended real valued, $\mathfrak{M}$ measurable function defined on $\boldsymbol{S}$ and assume that for each $B$ in $\mathfrak{B}$ it is true that $\int_{B} f(s) d \mu \leqq W B=\int_{X}{ }^{\prime} W(x, B) d \nu$. Such a function $f$ is said to be a lower bound function for the weights $W$-briefly, a l.b.f. $W$.

Lemma 4. Assume that $T$ is $B V W$ and that $f$ is a l.b.f. $W$. Assume that $N$ is a set in $\mathfrak{R}$ for which $\nu N=0$. Then $f(s)=0$ a.e. $\mu$ on $T^{-1} N$.

Proof. By A5 there is a set $A$ in $\mathfrak{A}$ such that $N \cong A$ and $\nu A=0$. Then $T^{-1} A$ is in $\mathfrak{B}$ and so

$$
\int_{T^{-1} \boldsymbol{A}} f(s) d \mu \leqq W T^{-1} A=\int_{\Lambda}{ }^{\prime} W\left(x, T^{-1} A\right) d \nu=0 .
$$

Theorem 5. Assume that $T$ is $B V W$ and that $f$ is a l.b.f. $W$. Suppose that $H$ is a real valued, $\mathfrak{R}$-measurable function defined on 
$X$. Then, for each $B$ in $\mathfrak{B}$, the function $H(x)^{\prime} W(x, B), x \in X$, is $\mathfrak{R}$ measurable and the function $H T(s) f(s), s \in B$, is M-measurable.

The proof is omitted. It resembles the proof of 2.13 in [6]. The following lemma is easy to check.

Lemma 6. Assume that $T$ is $B V W$ and that $f$ is a 1.b.f. $W$. such that $\int_{S} f(s) d \mu=W S$. Then $\int_{B} f(s) d \mu=W B$ for every $B$ in $\mathfrak{B}$.

Definition 7. Assume that $T$ is $B V W$ and that there exists a nonnegative, extended real valued function $f$ defined on $\boldsymbol{S}$ which is a 1.b.f. $W$ such that $\int_{S} f(s) d \mu=W S$. Then the transformation $T$ is said to be absolutely continuous with respect to the weights $W$-briefly, $T$ is $A C W$-and $f$ is termed a greatest lower bound function for the weights $W$-briefly, a g.l.b.f. $W$.

Observe that if $f$ is a g.l.b.f. $W$ then it follows from Lemma 6 that $\int_{B} f(s) d \mu=W B$ for every $B$ in $\mathfrak{B}$. It follows from A4 that $f$ is unique in the sense that any other g.l.b.f. $W$ must be equal to $f$ a.e. $\mu$ on $\boldsymbol{S}$.

THEOREM 8. Assume that $T$ is $B V W$. The following statements are equivalent.

(1) $T$ is $A C W$.

(2) The measure $W$ is absolutely continuous with respect to the measure $\mu \mid \mathfrak{B}$.

(3) If $B$ is a set in $\mathfrak{B}$ for which $\mu B=0$ then $W B=0$.

(4) If $B$ is a set in $\mathfrak{B}$ for which $\mu B=0$ then ${ }^{\prime} W(x, B)=0$ a.e. $\nu$ on $\boldsymbol{X}$.

Proof. That (2) implies (1) follows at once from the Radon-Nikodym Theorem. The other implications are obvious.

REMARK. Now assume in addition that $\nu T B=0$ whenever $B$ is any set in $\mathfrak{B}$ for which $\mu B=0$. Then $T$ is $A C W$ if and only if $T$ is $B V W$ (Use (4) above.).

3. The transformation formula. A basic formula for the transformation of integrals is established in this section. The standard hypotheses A1-A6 are assumed throughout the section.

Theorem 1. Assume that $T$ is $A C W$ and assume that $f$ is a g.l.b.f. $W$. Assume that $B$ is in $\mathfrak{B}$ and that $H$ is a real valued, $\mathfrak{N}$-measurable function defined on $\boldsymbol{X}$. Suppose that either $H^{\prime} W(., B)$ 
is ע-integrable over $\boldsymbol{X}$ or that $(H \circ T) f$ is $\mu$-integrable over $B$. Then

$$
\int_{X} H^{\prime} W(., B) d \nu=\int_{B}(H \circ T) f d \mu .
$$

Proof. The proof is quite similar to the proof of 4.10 in [6]. In fact, a careful study of the details of 4.7-4.10 of [6] reveals that, for the present discussion, it suffices to consider the case in which $H$ is the characteristic function of some set $N$ in $\mathfrak{R}$. Indeed it follows from A5 and 2.4 that we need only consider the case in which $H$ is the characteristic function of a set in $\mathfrak{A}$.

Thus assume that $H$ is the characteristic function $H_{A}$ of the set $A$ in 2 . It follows from 2.5 that $(H \circ T) f$ is $\mu$-integrable and that $H^{\prime} W(., B)$ is $\nu$-integrable. Evidently $\left(C T^{-1} A\right.$ denotes $\left.S-T^{-1} A\right)$

$$
\begin{aligned}
\int_{\boldsymbol{X}} H^{\prime} W(., & B) d \mu=\int_{X} H^{\prime} W\left(., B \cap T^{-1} A\right) d \nu+\int_{X} H^{\prime} W\left(., B \cap C T^{-1} A\right) d \nu \\
& =\int_{T^{\prime} B \cap A} H^{\prime} W\left(., B \cap T^{-1} A\right) d \nu+\int_{T^{B} B \cap O A} H^{\prime} W\left(., B \cap C T^{-1} A\right) d \nu \\
& =\int_{T^{\prime} B \cap A} W\left(., B \cap T^{-1} A\right) d \nu+0 \\
& =\int_{B \cap T^{-1} \boldsymbol{A}} f d \mu=\int_{B}(H \circ T) f d \mu
\end{aligned}
$$

Corollary 2. Assume that $T$ is $A C W$ and that $f$ is a g.l.b.f. $W$. Fix $B$ in $\mathfrak{B}$. Suppose that $H$ is a real valued, $\mathfrak{R}$-measurable function defined on $\boldsymbol{X}$ and assume that $H$ is bounded a.e. $\nu$ on TB. Then $H^{\prime} W(., B)$ is $\nu$-integrable over $\boldsymbol{X}$ and $(H \circ T) f$ is $\mu$-integrable over $B$. Moreover $\int_{X} H^{\prime} W(., B) d \nu=\int_{B}(H \circ T) f d \mu$.

LEMma 3. Let ' $W_{1}$ be a weighing function for $T$ and assume that $T$ is $A C W_{1}$ and that $W_{1} \leqq W$. Then for each $B$ in $\mathfrak{B}$ it is true that ' $W_{1}(x, B) \leqq{ }^{\prime} W(x, B)$ a.e. $\nu$ on $X$.

Proof. Let $f_{1}$ be a g.l.b.f. $W_{1}$. Fix $B$ in $\mathfrak{B}$ and let $A$ be any set in $\mathfrak{A}$. Then Theorem 1 and the properties of ' $W_{1}$ and ' $W$ lead to

$$
\begin{aligned}
\int_{\Delta}^{\prime} W_{1}(., B) d \nu & =\int_{B}\left(H_{\Delta} \circ T\right) f_{1} d \mu \\
& =\int_{B \cap T^{-1} \Delta} f_{1} d \mu=W_{1}\left(B \cap T^{-1} A\right) \\
& \leqq W\left(B \cap T^{-1} A\right)=\int_{T^{\prime} B \cap \Delta}{ }^{\prime} W\left(., B \cap T^{-1} A\right) d \nu \\
& \leqq \int_{\Delta}^{\prime} W(., B) d \nu .
\end{aligned}
$$


Theorem 4. Assume that $T$ is $A C W$ and that $f$ is a g.l.b.f. $W$. Assume that ' $W_{1}$ is a weighing function for $T$ and that $T$ is $A C W_{1}$. Suppose that $f_{1}$ is a g.l.b.f. $W_{1}$. Then $f_{1}(s) \leqq f(s)$ a.e. $\mu$ on $\boldsymbol{S}$ if and only if ' $W_{1}(x, B) \leqq{ }^{\prime} W(x, B)$ a.e. $\nu$ on $X$ for every $B$ in $\mathfrak{B}$.

Proof. Assume first that ' $W_{1}(x, B) \leqq{ }^{\prime} W(x, B)$ a.e. $\nu$ on $\boldsymbol{X}$ for every $B$ in $\mathfrak{B}$. Then $W_{1} \leqq W$ and so $\int_{B} f_{1}(s) d \mu \leqq \int_{B} f(s) d \mu$ for every $B$ in $\mathfrak{B}$. It follows from A4 that $f_{1}(s) \leqq f(s)$ a.e. $\mu$ on $S$. The opposite implication follows from .3.

Corollary 5. Assume that $T$ is $A C W$ and let $f$ be a g.1.b.f. $W$. Assume that ' $W_{1}$ is a weighing function for $T$, that $T$ is $A C W_{1}$, and that $f$ is also a g.l.b.f. $W_{1}$. Then ' $W_{1}(x, B)={ }^{\prime} W(x, B)$ a.e. $\nu$ on $\boldsymbol{X}$ for every $B$ in $\mathfrak{B}$.

This corollary implies that if two weighing functions ' $W$ and ' $W_{1}$ have the same weights $W=W_{1}$ and if $T$ is $A C W$ (and hence $\left.A C W_{1}\right)$ then the functions ' $W$ and ' $W_{1}$ are essentially the same.

4. A fundamental theorem. In this section a fundamental theorem is proved which gives a necessary and sufficient condition for an arbitrary nonnegative, real valued, $\mu$-integrable function $f$ to be a g.l.b.f. $W$ for some weighing function ' $W$. This result is presented in Theorem 8.

1. We introduce a new standard hypothesis.

A0. 2 is a $\sigma$-field of subsets $A$ of $\boldsymbol{X}$ having the following properties. $\mathfrak{A}$ is a subfamily of $\mathfrak{N}$ and $T^{-1} \mathfrak{A}$ is a subfamily of $\mathfrak{M}$. For each set $N$ in $\mathfrak{N}$ there is a set $A$ in $\mathfrak{A}$ such that $N \subseteq A$ and $\nu N=\nu A$. Also, (S) is a subfamily of $\mathfrak{M}$ with the property that $T \& S \subseteq \mathfrak{R}$.

Definition 2. Assume A0-A3. Let ' $W$ be a nonnegative, extended real valued function defined on $\boldsymbol{X} \times \mathfrak{M}$. ' $W$ is said to be a weighting function for $T$ if the following conditions are satisfied:

(i) If $G$ is in $\&$ then ' $W(., G)=0$ a.e. $\nu$ on $C T G$.

(ii) If a set $M$ in $\mathfrak{M}$ is the union of a countable number of pairwise disjoint sets $M_{i}$ in $\mathfrak{M}$ then ' $W(., M)=\Sigma^{\prime} W\left(., M_{i}\right)$ a.e. $\nu$ on $\boldsymbol{X}$.

(iii) For each $M$ in $\mathfrak{M}$ the function ${ }^{\prime} W(., M)$ is $\mathfrak{R}$-measurable (on $\boldsymbol{X})$.

Remark. Assume A1-A5. Then A0-A3 hold with (S) taken as $\mathfrak{B}$. If ' $W$ is a weighting function for $T$ then ' $W \mid \boldsymbol{X} \times \mathfrak{B}$ is a weighing function for $T$. 
Lemma 3. Assume A0-A3. There exists a set $A_{T}$ in $\mathfrak{X}$ such that $\nu A_{T^{\prime}}=0$ and such that for every $N$ in $\mathfrak{R}$ for which $\nu N=0$ it is true that $T^{-1}\left(N \cap C A_{T^{\prime}}\right)$ is in $\mathfrak{M}$ and $\mu T^{-1}\left(N \cap C A_{T^{\prime}}\right)=0$.

Proof. Define $\widetilde{C}^{\prime}$ to be the family of all sets $A$ in $\mathfrak{A}$ for which $\nu A=0$. By A1 the set $S$ can be expressed as the union of a countable number of sets $M_{n}, n \geqq 1$, in $\mathfrak{M}$ for which $\mu M_{n}<\infty, n \geqq 1$. For each positive integer $n$ let $\mathfrak{F}_{n}$ be the family of all sets of the form $T^{-1} A \cap M_{n}$ where $A$ is in $\mathbb{C}^{\prime}$. Each family $\mathbb{F}_{n}$ is a subfamily of $\mathfrak{M}$. For each $n \geqq 1$ set $P_{n}=\sup \left\{\mu M: M \in \mathfrak{\mho}_{n}\right\}$ and observe that $P_{n} \leqq$ $\mu M_{n}<\infty$. For each $n \geqq 1$ there is a sequence of sets $A_{n m}, m \geqq 1$, in (5' such that $\lim _{m} \mu\left(T^{-1} A_{n m} \cap M_{n}\right)=P_{n}$. Set $A_{T^{\prime}}=\bigcup_{n, m} A_{n m}$. Then $A_{T^{\prime}}$ is in (F' and $\mu\left(T^{-1} A_{T} \cap M_{n}\right)=P_{n}, n \geqq 1$.

To see that $A_{r}$ has the desired properties let $N$ be a set in $\mathfrak{N}$ for which $\nu N=0$. By A0 there is a set $A$ in $\mathfrak{A}$ such that $N \subseteq A$ and $\nu A=0$. For each $n$ it is true that

$$
\begin{aligned}
P_{n}= & \mu\left(T^{-1} A_{T^{\prime}} \cap M_{n}\right) \leqq \mu\left(T^{-1} A_{M} \cap M_{n}\right) \\
& +\mu\left[T^{-1}\left(A \cap C A_{T^{\prime}}\right) \cap M_{n}\right] \\
= & \mu\left[T^{-1}\left(A_{T^{\prime}} \cup\left(A \cap C A_{T^{\prime}}\right) \cap M_{n}\right] \leqq P_{n}<\infty .\right.
\end{aligned}
$$

Consequently $\mu T^{-1}\left(A \cap C A_{T^{\prime}}\right)=0$. According to $\mathrm{A} 1, T^{-1}\left(N \cap C A_{T^{\prime}}\right)$ is in $\mathfrak{M}$ and $\mu T^{-1}\left(N \cap C A_{T}\right)=0$.

Definition 4. Assume A0-A3. A set $A_{T}$ having the properties listed in the lemma is termed an essential $T$-set. (Hence, according to the lemma, there exists an essential $T$-set.)

If $A_{T}$ is an essential $T$-set and if $A$ is any set in $\mathfrak{U}$ for which $\nu A=0$ then $A_{T} \cup A$ is an essential $T$-set; hence, essential $T$-sets are by no means unique. However, if ${ }_{1} A_{T}$ and ${ }_{2} A_{T}$ are essential $T$-sets then $\mu\left(T^{-1}{ }_{1} A_{r} \Delta T^{-1}{ }_{2} A_{r}\right)=0$ (where $E \Delta F$ is the symmetric difference of the sets $E$ and $F$ ). Hence a function $f$ with domain $S$ vanishes a.e. $\mu$ on $T^{-1}{ }_{1} A_{T}$ if and only if it vanishes a.e. $\mu$ on $T^{-1}{ }_{2} A_{T}$.

THEOREM 5. Assume A0-A3. Let $A_{T}$ be an essential T-set. Let $f$ be a nonnegative, real valued, $\mu$-integrable function defined on $\boldsymbol{S}$. If $f(s)=0$ a.e. $\mu$ on $T^{-1} A_{T}$ then there exists a weighting function ' $W_{f}$ for $T$ which has the property that $\int_{M} f d \mu=\int_{X}{ }^{\prime} W_{f}(., M) d \nu$ for every $M$ in $\mathfrak{M}$.

Moreover the transformation formula holds for ' $W_{f}$ and $f$. More precisely, let $M$ be a set in $\mathfrak{M}$ and assume that $H$ is a real valued, $\mathfrak{R - m e a s u r a b l e ~ f u n c t i o n ~ d e f i n e d ~ o n ~} \boldsymbol{X}$. Suppose that either $H^{\prime} W_{f}(., M)$ 
is $\nu$-integrable over $\boldsymbol{X}$ or that $(H \circ T) f$ is $\mu$-integrable over $M . \quad$ Then $\int_{X} H^{\prime} W_{f}(., M) d \nu=\int_{\mu}(H \circ T) f d \mu$.

Proof. Assume $f(s)=0$ a.e. $\mu$ on $T^{-1} A_{T}$. Define a function $F$ on $\mathfrak{M}$ by $F(M)=\int_{M} f d \mu, M \in \mathfrak{M}$. $\quad F$ is a nonnegative, real valued measure on $\mathfrak{M}$. Now define for each $M$ in $\mathfrak{M}$ a nonnegative, real valued set function $\nu_{f_{M}}$ on $\mathfrak{A}$ by setting $\nu_{f_{M}} A=F\left(M \cap T^{-1} A\right)$ for every $A$ in $\mathfrak{A}$. Each of the functions $\nu_{f_{M}}$ is clearly a measure on $\mathfrak{A}$. Next it is shown that each of the measures $\nu_{f_{M}}$ is absolutely continuous with respect to the measure $\nu \mid \mathfrak{A}$. Thus fix a set $M$ in $\mathfrak{M}$ and let $A$ be a set in $\mathfrak{A}$ for which $\nu A=0$. Since $A_{T}$ is an essential $T$-set one finds that

$$
\begin{aligned}
\nu_{f_{M}} A=F\left(M \cap T^{-1} A\right) & =F\left(M \cap T^{-1} A \cap C T^{-1} A_{T}\right) \\
& \leqq F T^{-1}\left(A \cap C A_{T}\right)=0 .
\end{aligned}
$$

Hence for each $M$ in $\mathfrak{M}$ there is by the Radon-Nikodym Theorem a nonnegative, real valued, $\mathfrak{l}$-measurable function ' $W_{f}(., M)$ defined on $\boldsymbol{X}$ such that $\nu_{f_{M}} A=\int_{A}{ }^{\prime} W_{f}(., M) d(\nu \mid \mathfrak{A})=\int_{A}{ }_{A} W_{f}(., M) d \nu$ for every $A$ in 2 . Assert that ' $W_{f}$ is the desired weighting function for $T$. Clearly each function ' $W_{f}(., M)$ is $\mathfrak{R}$-measurable (on $\boldsymbol{X}$ ). Now fix $G$ in $\mathbb{E}$. By $\mathrm{A} 0$ there is a set $A$ in $\mathfrak{A}$ such that $C T G \subseteq A$ and $\nu(A \cap T G)=0$. Hence

$$
\begin{aligned}
\int_{\text {ort }}{ }^{\prime} W_{f}(., G) d \nu & =\int_{\Delta}{ }^{\prime} W_{f}(., G) d \nu=\nu_{f G} A \\
& =F\left(G \cap T^{-1} A\right)=F\left(G \cap T^{-1} A \cap T^{-1} C A_{T}\right)=0,
\end{aligned}
$$

since $G \cap T^{-1} A \cap T^{-1} C A_{T} \subseteq T^{-1}\left(T G \cap A \cap C A_{T}\right)$ and since $A_{T}$ is an essential $T$-set. Therefore ' $W_{f}(., G)=0$ a.e. $\nu$ on $C T G$. Next assume that the set $M$ in $\mathfrak{M}$ is the union of a countable number of pairwise disjoint sets $M_{i}$ in $\mathfrak{M}$. For every $A$ in $\mathfrak{A}$ it is clear that

$$
\int_{\Delta}^{\prime} W_{f}(., M) d \nu=\int_{\Delta} \Sigma^{\prime} W_{f}\left(., M_{i}\right) d \nu \text {. }
$$

Thus ' $W_{f}$ is a weighting function for $T$. Moreover if $M$ is in $\mathfrak{M l}$ then

$$
\int_{M} f d \mu=F\left(M \cap T^{-1} \boldsymbol{X}\right)=\nu_{f_{M}} \boldsymbol{X}=\int_{X}{ }_{X} W_{f}(., M) d \nu .
$$

Finally we prove that the transformation formula holds for ' $W_{f}$ and $f$. We first appeal to Definition 4 to deduce that $f(s)=0$ a.e. $\mu$ on $T^{-1} N$ whenever $N$ is a set in $\mathfrak{N}$ such that $\nu N=0$. Then observe that if we employ arguments entirely similar to those required in 3.1 it becomes clear that we need only consider the case in which $H$ is the 
characteristic function of a set $A$ in $\mathfrak{A}$. Thus fix $M$ in $\mathfrak{M}$ and let $H$ be the characteristic function of the set $A$ in $\mathfrak{A}$. We have

$$
\int_{\boldsymbol{X}} H^{\prime} W_{f}(., M) d \nu=\int_{\Delta}{ }^{\prime} W_{f}(., M) d \nu=F\left(M \cap T^{-1} A\right)=\int_{M}(H \circ T) f d \mu .
$$

(It must be emphasized that this transformation formula has been proved only for those particular weighting functions ' $W_{f}$ which arise in this proof. We have not proved a full analogue of 3.1.)

Corollary 6. Assume A0-A3. Let $f$ be a nonnegative, real valued, $\mu$-integrable function defined on $\boldsymbol{S}$. Assume $f(s)=0$ a.e. $\mu$ on $T^{-1} N$ whenever $N$ is any set in $\mathfrak{N}$ for which $\nu N=0$. Then there exists a weighting function ' $W_{f}$ for $T$ which has the property that $\int_{M} f d \mu=\int_{x}{ }^{\prime} W_{f}(., M) d \nu$ for every $M$ in $\mathfrak{M}$. Moreover the transformation formula holds for ' $W_{f}$ and $f$ (cf. Theorem 5).

Corollary 7. Assume A1-A5. Let $A_{T}$ be an essential T-set. Let $f$ be a nonnegative, real valued, $\mu$-integrable function defined on $\boldsymbol{S}$. Then there exists a weighing function ' $W_{f}$ for $T$ for whose weights $f$ is a greatest lower bound function if and only if $f(s)=0$ a.e. $\mu$ on $T^{-1} A_{T}$.

THEOREM 8. Assume A1-A5. Let $f$ be a nonnegative, real valued, $\mu$-integrable function defined on $\boldsymbol{S}$. Then there exists a weighing function ' $W_{f}$ for $T$ for whose weights $f$ is a greatest lower bound function if and only if $f=0$ a.e. $\mu$ on $T^{-1} N$ whenever $N$ is any set in $\mathfrak{R}$ for which $\nu N=0$.

The rest of the section is concerned with a "Lebesgue decomposition" theorem for weighing functions. Rather similar theorems for weight functions appear in [1].

Definition 9. Assume A1-A6 and assume that ' $W_{0}$ is a weighing function for $T$ having the properties that ' $W_{0} \leqq{ }^{\prime} W$ and that $T$ is $A C W_{0}$. Then ' $W_{0}$ is termed an $A C$ part of ' $W$.

Definition 10. Assume A1-A6. Assume that ' $W_{0}$ is an $A C$ part of ' $W$. Then ' $W$ is said to be a maximal $A C$ part of ' $W$ in case it is true that whenever ' $W_{1}$ is any $A C$ part of ' $W$ then, for each $B$ in $\mathfrak{B}$, one has ' $W_{1}(x, B) \leqq{ }^{\prime} W_{0}(x, B)$ a.e. $\nu$ on $\boldsymbol{X}$. ' $W$ is said to be trivial in case ' $W(x, B)=0$ a.e. $\nu$ on $\boldsymbol{X}$ for every $B$ in $\mathfrak{B}$. (' $W$ is trivial if and only if $W$ vanishes identically on $\mathfrak{B}$.) ' $W$ is said to be singular if all of its $A C$ parts are trivial and if there also exists a set $B_{0}$ in $\mathfrak{B}$ 
such that $\mu B_{0}=0$ and such that for each $B$ in $\mathfrak{B}$ one has ' $W(x, B)=0$ a.e. $\nu$ on $C T B_{0}$. Assume that ' $W_{A}$ and ' $W_{S}$ are weighing functions for $T$. The ordered pair $\left({ }^{\prime} W_{A},{ }^{\prime} W_{S}\right)$ is termed a Lebesgue-type decomposition for ' $W$ if ' $W={ }^{\prime} W_{A}+{ }^{\prime} W_{S}$, if ' $W_{A}$ is a maximal $A C$ part of ' $W$, and if ' $W_{S}$ is singular.

Note that if $T$ is $B V W$ then it follows from the above definitions that ' $W$ can have essentially at most one Lebesgue-type decomposition in the following sense: If $\left({ }^{\prime} W_{A},{ }^{\prime} W_{S}\right)$ and $\left({ }^{\prime} W_{a},{ }^{\prime} W_{s}\right)$ are Lebesgue-type decompositions for ' $W$ then ${ }^{\prime} W_{\boldsymbol{A}}(., B)={ }^{\prime} W_{a}(., B)$ and ${ }^{\prime} W_{S}(., B)={ }^{\prime} W_{s}(., B)$ a.e. $\nu$ on $X$ for every $B$ in $\mathfrak{B}$.

Theorem 11. Assume A1-A6 and assume that $T$ is $B V W$. Then 'W has one (and essentially only one) Lebesgue-type decomposition.

Proof. By the Lebesgue Decomposition Theorem for measures [4, p. 132] there exist nonnegative, real valued measures $\mu_{\Delta}$ and $\mu_{S}$ defined on $\mathfrak{B}$ such that $W=\mu_{A}+\mu_{S}$, such that $\mu_{\Delta}$ is absolutely continuous with respect to $\mu / \mathfrak{B}$, and such that $\mu_{S}$ is $\mu / \mathfrak{B}$-singular. According to the Radon-Nikodym Theorem there is a nonnegative, $\mathfrak{B}$-measurable function $f$ defined on $S$ such that $\mu_{\mathbb{A}} B=\int_{B} f(s) d(\mu / \mathfrak{B})=\int_{B} f(s) d \mu$ for every $B$ in $\mathfrak{B}$. Let $A_{T}$ be an essential $T$-set (see Definition 4 ). It follows from 2.4 that $f(s)=0$ a.e. $\mu$ on $T^{-1} A_{T}$. By 7 . there is a weighing function ' $W_{a}$ for $T$ for whose weights $W_{a} f$ is a g.l.b.f. $W_{a}$. Hence $W_{a}=\mu_{A} \leqq W$. It follows from 3.3 that ' $W_{a}(x, B) \leqq{ }^{\prime} W(x, B)$ a.e. $\nu$ on $X$ for every $B$ in $\mathfrak{B}$. For each $B$ there is a set $X_{B}$ in $\mathfrak{R}$ such that $\nu X_{B}=0$ and such that ' $W_{a}(x, B) \leqq ' W(x, B)<\infty$ whenever $x$ is in $C X_{B}$. Define nonnegative, extended real valued functions ' $W_{A}$ and ' $W_{S}$ on $X \times \mathfrak{B}$ as follows: For each $B$ in $\mathfrak{B}$ set ' $W_{\Lambda}(x, B)={ }^{\prime} W_{a}(x, B)$ and ' $W_{S}(x, B)=$ ' $W(x, B)-{ }^{\prime} W_{a}(x, B)$ if $x$ is in $C X_{B}$ and put ' $W_{A}(x, B)=0$ and ' $W_{S}(x, B)={ }^{\prime} W(x, B)$ if $x$ is in $X_{B}$. It is easy to verify that both ' $W_{A}$ and ' $W_{S}$ are weighing functions for $T$.

It is now shown that $\left({ }^{\prime} W_{A},{ }^{\prime} W_{S}\right)$ is a Lebesgue-type decomposition for ' $W$. Clearly ' $W={ }^{\prime} W_{A}+{ }^{\prime} W_{s}$. Moreover ' $W_{A}$ is an $A C$ part of ' $W$. Let ' $W_{0}$ be any $A C$ part of ' $W$. Let $f_{0}$ be a g.l.b.f. $W_{0}$ and observe that $f$ is a g.l.b.f. $W_{\boldsymbol{A}}$. Define a function on $\boldsymbol{S}$ by setting $f *=\max \left\{f, f_{0}\right\}$. According to Corollary 7 there is a weighing function ' $W *$ for whose weights $W * f *$ is a greatest lower bound function. Consider the set $M *$ of points $s$ in $\boldsymbol{S}$ for which $f_{0}(s)>f(s) . \quad M *$ is in $\mathfrak{M}$ and so there is a set $B *$ in $\mathfrak{B}$ such that $M * \subseteq B *$ and $\mu(B * \cap C M *)=0$. It is clear that for every $B$ in $\mathfrak{B}$ one has

$$
W * B=W_{0}(B \cap B *)+W_{A}(B \cap C B *) \leqq W B .
$$

Hence $W_{A} \leqq W * \leqq W$. The function $W_{m}=W *-W_{A}$ is a measure on 
$\mathfrak{B}$ and, in view of $2.8, W_{m}$ is absolutely continuous with respect to $\mu / \mathfrak{B}$. Note that $W_{m} \leqq W_{S}=\mu_{S}$ and so $W_{m}$ is also $\mu / \mathfrak{B}$-singular. Hence $W *=W_{\boldsymbol{A}}$. It follows that $f(s) \geqq f_{0}(s)$ a.e. $\mu$ on $\boldsymbol{S}$ so that, in view of $3.4,{ }^{\prime} W_{0}(x, B) \leqq{ }^{\prime} W_{A}(x, B)$ a.e. $\nu$ on $X$ for every $B$ in $\mathfrak{B}$. It follows from Definition 10 that ' $W_{A}$ is a maximal $A C$ part of ' $W$. Finally it is shown that ' $W_{S}$ is singular. Since $\mu_{S}$ is $\mu / \mathfrak{B}$-singular there is a set $B_{0}$ in $\mathfrak{B}$ such that $\mu B_{0}=0$ and $W_{S}\left(C B_{0}\right)=\mu_{S}\left(C B_{0}\right)=0$. Given $B$ in $\mathfrak{B}$, one obtains

$$
\begin{aligned}
\int_{\sigma^{T} B_{0}}{ }^{\prime} W_{S}(., B) d \nu= & \int_{\sigma^{T} B_{0}}{ }^{\prime} W_{S}\left(., B \cap B_{0}\right) d \nu \\
& +\int_{\sigma^{T} B_{0}}{ }^{\prime} W_{S}\left(., B \cap C B_{0}\right) d \nu \leqq 0+W_{S}\left(C B_{0}\right)=0 ;
\end{aligned}
$$

hence ' $W_{s}(x, B)=0$ a.e. $\nu$ on $C T B_{0}$. It is clear that ' $W_{s}$ can have no nontrivial $A C$ parts.

5. Connection with earlier work. The relationship between the transformation theory of [6] and the theory just presented is discussed briefly in this section. The results in [6] are obtained in a setting which is established by imposing the standard hypotheses H1-H8 (see 1 of [6]). Other hypotheses-denoted by H9-H15-are imposed in certain situations in [6]. Still others have been introduced in subsequent papers; one of these-HC1 (see [2])-is of particular interest in the present discussion. For if it is assumed that H1-H8 and $\mathrm{HC} 1$ are in effect then it is easy to verify that A1-A5 are also satisfied; of course it is necessary to replace $S^{\prime}$ by $\boldsymbol{X}$, $\mathfrak{M}^{\prime}$ by $\mathfrak{R}, \mu^{\prime}$ by $\nu$, and $\mathfrak{B}^{\prime}$ by $\mathfrak{A}$ throughout the statements of $\mathrm{H} 1-\mathrm{H} 8$ and HC1. Hence if $\mathrm{H} 1-\mathrm{H} 8$ and $\mathrm{HC1}$ are assumed to hold then both of the theories apply. Thus within this context the concepts of weight function and weighing function can be compared. Moreover it is clear that if $\mathrm{H} 1-\mathrm{H} 8$ are in effect then A0-A3 hold, provided that one makes the replacements indicated above and puts $\mathbb{B S}=\mathfrak{D}$.

The desired comparison of weighing functions and weight functions requires a modification of $\mathrm{H} 9$ (and hence the comparison is rather indirect). The modified statement is denoted by $\mathrm{H} 9 *$.

$\mathrm{H} 9 * . \quad W^{\prime}$ is a nonnegative, extended real valued function defined on $\boldsymbol{X} \times \mathfrak{D}$ and satisfying the following conditions.

(i)* If $D$ is in $\mathscr{D}$ then $W^{\prime}(x, D)=0$ a.e. $\nu$ on $C T D$.

(ii)* If a set $D$ in $D$ contains a countable number of pairwise disjoint sets $D_{i}$ in $\mathfrak{D}$ then $\sum W^{\prime}\left(x, D_{i}\right) \leqq W^{\prime}(x, D)$ a.e. $\nu$ on $\boldsymbol{X}$.

(iii)* If a set $D$ in $\mathfrak{D}$ is the union of a sequence of sets $D_{j}$ in (D) for which $D_{j} \subseteq D_{j+1}$ for every $j$ then $\lim W^{\prime}\left(x, D_{j}\right)=W^{\prime}(x, D)$ a.e. $\nu$ on $X$.

(iv)* For each $D$ in $\mathfrak{D}$ the function $W^{\prime}(., D)$ is $\Re$-measurable (on $\boldsymbol{X}$ ). 

for $T$.

A function having these properties is termed a quasiweight function

Remarks 1. Assume H1-H8. Every weight function for $T$ is a quasi-weight function for $T$. But a quasi-weight function may not be a weight function. It is easy to verify that the results in $2,3,4,5$, and 6.1 of [6] remain valid if $\mathrm{H} 9$ is replaced by $\mathrm{H} 9 *$ throughout those sections; the notational changes indicated above must be kept in mind.

Remarks 2. Assume $\mathrm{H} 1-\mathrm{H} 8$ and $\mathrm{HC1}$. It is easy to see that if ' $W$ is a weighing function for $T$ then the function $W^{\prime} \equiv{ }^{\prime} W \mid \boldsymbol{X} \times \mathfrak{D}$ satisfies $\mathrm{H} 9 *$. Hence the function ' $W \mid \boldsymbol{X} \times \mathfrak{D}$ is a quasi-weight function for $T$ and so, for example, it may play the role of $W^{\prime}$ in the results of $2,3,4,5$, and 6.1 in [6]. Thus the arbitrary weighing function ' $W$ gives rise in a natural manner to the quasi-weight function ' $W \mid \boldsymbol{X} \times \mathfrak{D}$. The next theorem shows that, conversely, certain quasi-weight functions are restrictions (to $\boldsymbol{X} \times \mathfrak{D}$ ) of weighing functions.

THEOREM 3. Assume $\mathrm{H} 1-\mathrm{H} 8, \mathrm{H} 9 *$, and $\mathrm{HC1}$ and suppose that there is a nonnegative, real valued, $\mu$-integrable function $f$ defined on $\boldsymbol{S}$ for which $\int_{D} f(s) d \mu=\int_{X} W^{\prime}(x, D) d \nu$ for every $D$ in $\mathfrak{D}$. Then there exists a weighing function ' $W$ for $T$ for which $W^{\prime}(x, D)=$ ' $W(x, D)$ for every $(x, D)$ in $\boldsymbol{X} \times \mathfrak{D}$. Moreover $f$ is a greatest lower bound function for the weights induced by ' $W$.

Proof. The lemma in 2.6 [6] applies here even though H9 has been dropped in favor of $\mathrm{H} 9 *$. Thus if $A_{r}$ is an essential $T$-set it follows that $f(s)=0$ a.e. $\mu$ on $T^{-1} A_{T}$. According to 4.7 there is a weighing function ' $W_{0}$ for $T$ for whose weights $f$ is a greatest lower bound function. Fix $D$ in $\mathscr{D}$ and let $N$ be in $\mathfrak{R}$. The results in 4.8 of [6] (with $\mathrm{H} 9$ replaced by $\mathrm{H} 9 *$ ) and in 3.1 lead to

$$
\int_{N} W^{\prime}(., D) d \nu=\int_{D}\left(H_{N} \circ T\right) f d \mu=\int_{N}{ }^{\prime} W_{0}(., D) d \nu .
$$

Therefore $W^{\prime}(x, D)={ }^{\prime} W_{0}(x, D)$ a.e. $\nu$ on $\boldsymbol{X}$.

Hence for each $D$ in $\mathfrak{D}$ there is a set $X_{D}$ in $\mathfrak{R}$ such that $\nu X_{D}=0$ and such that $W^{\prime}(x, D)={ }^{\prime} W_{0}(x, D)$ if $x$ is not in $X_{D}$. For a set $B$ which is in $\mathfrak{B}$ but not in $\mathfrak{D}$ set $X_{B}=\varnothing$. Then define a nonnegative, extended real valued function ' $W$ on $\boldsymbol{X} \times \mathfrak{B}$ as follows: For each $B$ in $\mathfrak{B}$ put ' $W(x, B)={ }^{\prime} W_{0}(x, B)$ if $x$ is in $C X_{B}$ and set ' $W(x, B)=W^{\prime}(x, B)$ if $x$ is in $X_{B}$. Evidently ' $W$ is the desired weighing function for $T$.

Corollary 4. Assume $\mathrm{H} 1-\mathrm{H} 9$ and $\mathrm{HC1}$ and assume that $T$ is 
$A C W$ (see 3.4 of [6]). Then there exists a weighing function ' $W$ for $T$ for which ' $W(x, D)=W^{\prime}(x, D)$ for every $(x, D)$ in $\boldsymbol{X} \times \mathfrak{D}$. Moreover the weights induced by ' $W$ and $W$ ' have the same greatest lower bound function.

Remarks 5. Assume H1-H8. It is easy to see that if ' $W$ is a weighting function for $T$ then the function ${ }^{\prime} W \mid \boldsymbol{X} \times \mathfrak{D}$ is a quasiweight function for $T$ (cf. Remarks 2).

TheOREm 6. Assume H1-H8 and $\mathrm{H} 9 *$ and suppose that there is a nonnegative, real valued, $\mu$-integrable function $f$ defined on $\boldsymbol{S}$ for which $\int_{D} f d \mu=\int_{X} W^{\prime}(., D) d \nu$ for every $D$ in $\mathfrak{D}$. Then there exists a weighting function ' $W$ for $T$ for which $W^{\prime}(x, D)={ }^{\prime} W(x, D)$ for every $(x, D)$ in $\boldsymbol{X} \times \mathfrak{D}$. Moreover $\int_{M} f d \mu=\int_{X}{ }^{\prime} W(., M) d \nu$ for every $M$ in $\mathfrak{M}$ and (cf. 4.5) the transformation formula holds for ' $W$ and $f$.

As one would expect, the proof of this theorem parallels that of Theorem 3, with 4.5 now playing the roles of 4.7 and 3.1 in the earlier argument.

Corollary 7. Assume H1-H9. Assume that $T$ is $A C W$ and let $f$ be a g.l.b.f. $W$. Then there exists a weighting function ' $W$ for $T$ for which ' $W(x, D)=W^{\prime}(x, D)$ for every $(x, D)$ in $\boldsymbol{X} \times \mathfrak{D}$. Moreover $\int_{M} f d \mu=\int_{X}{ }^{\prime} W(., M) d \nu$ for every $M$ in $\mathfrak{M}$ and $(c f .4 .5)$ the transformation formula holds for ' $W$ and $f$.

Remarks 8. Assume H1-H8, HC1, and H11-H12 (see [6]). Consider the family $\leftleftarrows$ which was introduced in 7.6 in [6]. One can verify that, in view of $\mathrm{H} 12$, the standard hypothesis $\mathrm{HC} 1$ remains satisfied even if $\mathfrak{B}$ is replaced by $\mathfrak{B} \cap \mathfrak{F}$. If this replacement is made then 4.7 of [2] and 7.8 of [6] lead to the following conclusion:

Assume $\mathrm{H} 1-\mathrm{H} 8, \mathrm{H} 11-\mathrm{H} 13, \mathrm{HC} 1$, and $\mathrm{H} 15 \mathrm{i}$ and assume that $T$ is $B V W$. Then the function $W_{*}^{\prime}(x, B, w),(x, B) \in \boldsymbol{X} \times \mathfrak{B}$, is a weighing function for T. (See [2] for a statement of H15i and see [6] for H13. The expression $W_{*}^{\prime}(x, B, w)$ is defined in 7.7 of [6].)

REMARKS 9. The preceding discussion has dealt only with the relationship between the transformation theory presented in [6] and the present study. For a discussion of the relationship between [6] and earlier work in the subject the reader is advised to see 9 of [6].

Finally it should be noted that in a forthcoming paper the author will discuss the problem of obtaining analogues for 4.7 and 4.8 within the context set forth in [6]. 
The author wishes to express his gratitude to Prof. P. V. Reichelderfer and to the referee for suggesting several improvements.

\section{BIBLIOGRAPHY}

1. R. W. Chaney, Decomposition theorems for weight functions in the transformation theory for measure space (to appear in Rendiconti del Circolo Matematico di Palermo) 2. Measurability theorems in the transformation theory for measure space (to appear in Rendiconti del Circolo Matematico di Palermo)

3. G. A. Craft, A transformation theory for weight functions, Rendiconti del Circolo Matematico di Palermo 10 (1961), 212-228.

4. N. Dunford and J. T. Schwartz, Linear Operators, Part 1: General Theory, Interscience, New York, 1958.

5. T. Rado and P. Reichelderfer, Continuous Transformations in Analysis, SpringerVerlag, Berlin, Göttingen, Heidelberg, 1955.

6. P. Reichelderfer, A transformation theory for measure space, Rendiconti del Circolo Matematico di Palermo 10 (1961), 283-313.

7. S. Saks, Theory of the Integral, second revised edition, Monografie Matematyczne, Vol. 7, Warszawa-Lwow, 1937.

Received August 19, 1965.

Western Washington State College, Bellingham 


\title{
PACIFIC JOURNAL OF MATHEMATICS
}

\author{
EDITORS
}

\section{H. SAMELSON}

Stanford University

Stanford, California

J. P. JANS

University of Washington

Seattle, Washington 98105
J. DugundJI

University of Southern California

Los Angeles, California 90007

RICHARD ARENS

University of California

Los Angeles, California 90024

\section{ASSOCIATE EDITORS}

\section{E. F. BECKENBACH}

B. H. NEUMANN

F. WOLF

K. YosidA

\section{SUPPORTING INSTITUTIONS}

\author{
UNIVERSITY OF BRITISH COLUMBIA \\ CALIFORNIA INSTITUTE OF TECHNOLOGY \\ UNIVERSITY OF CALIFORNIA \\ MONTANA STATE UNIVERSITY \\ UNIVERSITY OF NEVADA \\ NEW MEXICO STATE UNIVERSITY \\ OREGON STATE UNIVERSITY \\ UNIVERSITY OF OREGON \\ OSAKA UNIVERSITY \\ UNIVERSITY OF SOUTHERN CALIFORNIA
}

\author{
STANFORD UNIVERSITY \\ UNIVERSITY OF TOKYO \\ UNIVERSITY OF UTAH \\ WASHINGTON STATE UNIVERSITY \\ UNIVERSITY OF WASHINGTON \\ AMERICAN MATHEMATICAL SOCIETY \\ CHEVRON RESEARCH CORPORATION \\ TRW SYSTEMS \\ NAVAL ORDNANCE TEST STATION
}

Mathematical papers intended for publication in the Pacific Journal of Mathematics should be typewritten (double spaced). The first paragraph or two must be capable of being used separately as a synopsis of the entire paper. It should not contain references to the bibliography. Manuscripts may be sent to any one of the four editors. All other communications to the editors should be addressed to the managing editor, Richard Arens at the University of California, Los Angeles, California 90024.

50 reprints per author of each article are furnished free of charge; additional copies may be obtained at cost in multiples of 50 .

The Pacific Journal of Mathematics is published monthly. Effective with Volume 16 the price per volume (3 numbers) is $\$ 8.00$; single issues, $\$ 3.00$. Special price for current issues to individual faculty members of supporting institutions and to individual members of the American Mathematical Society: $\$ 4.00$ per volume; single issues $\$ 1.50$. Back numbers are available.

Subscriptions, orders for back numbers, and changes of address should be sent to Pacific Journal of Mathematics, 103 Highland Boulevard, Berkeley 8, California.

Printed at Kokusai Bunken Insatsusha (International Academic Printing Co., Ltd.), No. 6, 2-chome, Fujimi-cho, Chiyoda-ku, Tokyo, Japan.

PUBLISHED BY PACIFIC JOURNAL OF MATHEMATICS, A NON-PROFIT CORPORATION

The Supporting Institutions listed above contribute to the cost of publication of this Journal, but they are not owners or publishers and have no responsibility for its content or policies. 


\section{Pacific Journal of Mathematics \\ Vol. 19, No. $2 \quad$ June, 1966}

Leonard Daniel Baumert, Extreme copositive quadratic forms . ......... 197

Fred James Bellar, Jr., Pointwise bounds for the second initial-boundary value problem of parabolic type ........................ 205

L. Carlitz and David Paul Roselle, Restricted bipartite partitions ........ 221

Robin Ward Chaney, On the transformation of integrals in measure space ........................................... 229

Colin W. Clark, An embedding theorem for function spaces ........... 243

Edwin Duda, A theorem on one-to-one mappings................ 253

Ben Fitzpatrick, Jr. and Donald Reginald Traylor, Two theorems on metrizability of Moore spaces.......................... 259

Allen Roy Freedman, An inequality for the density of the sum of sets of vectors in $n$-dimensional space ....................... 265

Michael Friedberg, On representations of certain semigroups .......... 269

Robert William Gilmer, Jr., The pseudo-radical of a commutative ring . . . . 275

Hikosaburo Komatsu, Fractional powers of operators ............... 285

Daniel Rider, Transformations of Fourier coefficients ................. 347

David Alan Sánchez, Some existence theorems in the calculus of variations ...................................... 357

Howard Joseph Wilcox, Pseudocompact groups............... 365

William P. Ziemer, Some lower bounds for Lebesgue area ............. 381 